\title{
Adoption of Telemedicine During the COVID-19 Pandemic: Perspectives of Primary Healthcare Providers
}

\author{
Joe Lintz ${ }^{1 *}$ (1) \\ ${ }^{1}$ Health Information Management Department, Parker University, Dallas, TX, USA \\ *Corresponding Author: cclintz@hotmail.com \\ Citation: Lintz, J. (2022). Adoption of Telemedicine During the COVID-19 Pandemic: Perspectives of Primary Healthcare Providers. European \\ Journal of Environment and Public Health, 6(1), em0106. https://doi.org/10.21601/ejeph/11804
}

\section{ARTICLE INFO}

Received: 2 Sep. 2021

Accepted: 8 Feb. 2022

\begin{abstract}
Background: As the pandemic has unfolded across an array of communities worldwide, telemedicine has been promoted and scaled up to attend to and reduce person-to-person transmission of COVID-19 in the U.S. and internationally. This study examined the major barriers to adoption of telemedicine among primary healthcare providers at a primary care clinic in north Texas during the pandemic.

Methods: A self-administered questionnaire was mailed to 67 primary healthcare providers at a primary care clinic in north Texas, with a 70\% percent response rate $(n=47)$. The survey collected information on perceived barriers to telemedicine adoption and experience with telemedicine in the last 12 months. In addition to descriptive statistics, multiple logistic regression was conducted to determine characteristics related to use of telemedicine in the past year. Chi-square tests were also performed to examine the relationship between the number of perceived barriers and telemedicine usage.

Results: Results revealed that the lack of reimbursement was a significant obstacle to telemedicine adoption. Plus, the number of perceived barriers to telemedicine usage was negatively associated with using telemedicine.

Conclusions: The findings suggested that the barriers to using telemedicine existed across an array of situations and that decreasing these obstacles would be critical in encouraging future telemedicine adoption among providers during and after the pandemic. This will especially be the case for primary care practices where scarce financial resources have been a traditional problem for such providers. This complicacy is likely to be amplified owing to the inimical effects of the pandemic, during and after its potential containment or successful management of it.
\end{abstract}

Keywords: telemedicine adoption, implementation, COVID-19, pandemic, primary healthcare providers

\section{INTRODUCTION}

The coronavirus 2019 (COVID-19) pandemic presents an unprecedented threat in the global community with over $114,853,685$ cases reported in 223 countries as of 4 th March 2021 (World Health Organization-WHO, n.d.; WHO, 2020). As the pandemic unfolded worldwide, telemedicine-particularly video consultations-has been promoted and scaled up to attend to and reduce person-to-person transmission of COVID-19 in the U.S. and internationally (Ohannessian et al., 2020). Telemedicine refers to:

The exchange of medical information from one site to another through electronic communications to improve a patient's health. The electronic communications consist of using real-time interactive video visits and shared diagnostic images, vital signs, and video clips (Centers for Medicare \& Medicaid Services-CMS, 2020: 1).

Previous empiricism has shown that increased employment of telemedicine services has played a critical and beneficial role in previous coronavirus outbreaks, such as SARS (severe acute respiratory syndrome), MERS (Middle East respiratory syndrome), and Ebola and Zika viruses (Ohannessian et al., 2020). The most important aspect of telemedicine is its ability to enable patients to connect with their healthcare provider at a distance. This would lead to a substantial decrease in unnecessary patients visits, promoting self-quarantine, and reducing emergency department overuse during the COVID-19 pandemic (Moazzami et al., 2020; Rahi et al., 2020). However, adoption of telemedicine in response to COVID-19 remains a challenge for many providers (Kruse et al., 2018; Ohannessian et al., 2020). These challenges include absence of a regulatory framework to allow integration of and sufficient reimbursement for telemedicine for all patients, 
particularly in emergency and outbreak situations (Ohannessian et al., 2020). Providers in small- to mediumsized facilities-such as primary care clinics and specialty practices-are not equipped to deliver care via telemedicine owing to a lack of sufficient IT budgets and other resources (Blue et al., 2019; Hing et al., 2008; Levine et al., 2014; Lintz, 2020; Martin et al., 2012). Therefore, many patients are unlikely to be recipients of the salutary outcomes telemedicine engenders.

Moreover, a systematic review of 30 articles addressing adoption of telemedicine among healthcare providers discerned that the major impediments were related to cost and reimbursement aspects, followed by absence of integration and technical coordination across multiple organizations (Kruse et al., 2018). For example, early studies have found that costs associated with adoption of telemedicine and lack of adequate reimbursement for telemedicine services are a significant challenge that states, counties, and providers face in implementing telemedicine (Kruse et al., 2018; Molfenter, 2015). More recent findings have further suggested that this dilemma continues. In addition, despite the growth of telemedicine services during the pandemic, scholars have shown that obtaining technical support for the requisite electronic infrastructure was a significant barrier to adoption of telemedicine (Kruse et al., 2018; Levine et al., 2014; Sisk et al., 2020).

\section{Statement of the Problem}

Existing empiricism has shown that a paucity of financial resources, inconsistencies with reimbursement, and inability to gather and transmit seemingly limitless amounts of data are the most significant barriers contributing to primary healthcare providers' difficulty to provide care via telemedicine (Blue et al., 2019; Bodenheimer and Pham, 2010; Kruse et al., 2018; Lintz, 2020). Owing to this ill-preparedness of certain primary healthcare providers to implement telemedicine, some patients might not receive timely diagnosis and treatment through telemedicine efforts that may well assist in decreasing human exposure to COVID-19 while ensuring that medical services are available for patients critically needing them. A primary healthcare provider in the current study is defined as a primary specialty of general practice or family practice medicine, per the Texas Department of State Health Services (TDSHS) (Lintz, 2020).

\section{Purpose of the Study and Research Questions}

The purpose of this study was to examine the major barriers to adoption of telemedicine among primary healthcare providers at a primary care clinic in north Texas during the pandemic. In addition, the research gleaned information about respondent characteristics vis-a-vis telemedicine use.

The study was designed to answer the following research questions (RQ):

1. What are the perceived barriers to adoption of telemedicine among primary healthcare providers in large urban areas?

2. What provider characteristics (e.g., age and length of service) are associated with using telemedicine during the pandemic in large urban areas?
3. Is there a significant relationship between the number of perceived barriers to adoption of telemedicine and its usage in large urban areas in the past 12 months?

Justification for investigating these three issues will be subsequently provided.

Despite the fact that the recent policies have expanded coverage for telemedicine payment during the COVID-19 pandemic, the extent to which barriers to successful adoption and implementation can be identified and remedied among certain primary healthcare providers remains unclear (CMS, 2020; Sisk et al., 2020). Furthermore, previous research suggested that provider characteristics and perceptions of barriers to telemedicine adoption likely play a significant role in determining telemedicine usage rates (CMS, 2020; Sisk et al., 2020). Hence, the current research questions were designed to answer these questions by understanding the barriers and characteristics associated with telemedicine adoption among providers during this public health crisis (Kruse et al., 2018; Redd et al., 2015).

\section{Significance of the Study}

Notwithstanding more recent regulations have improved reimbursements for telemedicine services (Chauhan et al., 2020; Friedman et al., 2019; Paschall, n. d.; Smith et al., 2019), little is known about the extent to which barriers are unique to successful adoption of telemedicine have been identified, or possible public policy alternatives to overcoming those barriers during the pandemic from primary healthcare providers' perspectives. Thus, the present investigation could provide invaluable information about principal obstacles that primary healthcare providers encounter while seeking to increase their use of telemedicine to help manage patient populations effectively during the pandemic. Additionally, the outcome of the study might serve as a beneficial information resource for state and local public health agencies as they seek to promote use of telemedicine during the COVID-19 public health emergency and to determine if gains in telemedicine use will continue postpandemic (Paschall, n. d.).

\section{Theoretical Framework for the Study}

Based on the literature review, the present study utilized a well-known conceptual model of perceived major barriers to provider telemedicine usage (Primary Care Collaborative, 2020). According to this model, although providers were using more telehealth, major concerns remained regarding payment, regulations, and technical issues concerning these services (Primary Care Collaborative, 2020). The studies of this theoretical framework in the literature provide an understanding of the major impediments to telehealth adoption among providers, as well as provider characteristics associated with using telehealth. Therefore, this work's theoretical framework appears to be well suited for the current research vis-à-vis evaluating and analyzing the major obstacles to adoption of telemedicine among primary healthcare providers in response to the COVID-19 pandemic, as well as provider characteristics related to telemedicine use. 
Table 1. Characteristics of the respondents and percentage of the respondents mentioned barriers to telemedicine adoption $(n=47)$

\begin{tabular}{|c|c|c|}
\hline Characteristics & $\begin{array}{l}\text { Number of } \\
\text { respondents }\end{array}$ & Percentage \\
\hline \multicolumn{3}{|l|}{ Gender } \\
\hline Male & 28 & 60 \\
\hline Female & 19 & 40 \\
\hline \multicolumn{3}{|l|}{ Age } \\
\hline 39 years or younger & 27 & 57 \\
\hline 40-49 years & 10 & 21 \\
\hline 50-59 years & 7 & 15 \\
\hline $60+$ years & 3 & 7 \\
\hline \multicolumn{3}{|l|}{ Location } \\
\hline Urban & 28 & 60 \\
\hline Suburban & 19 & 40 \\
\hline \multicolumn{3}{|l|}{ Years of employment } \\
\hline Less than 5 year & 6 & 13 \\
\hline $5-9$ years & 10 & 21 \\
\hline $10-14$ years & 20 & 43 \\
\hline 15 years or more & 11 & 23 \\
\hline \multicolumn{3}{|l|}{ Subspecialist status } \\
\hline Primary care & 38 & 80 \\
\hline Subspecialist & 9 & 20 \\
\hline \multicolumn{3}{|l|}{ Barriers to telemedicine adoption } \\
\hline Cost of equipment & & 30 \\
\hline Lack of reimbursement & & 28 \\
\hline Inadequate technical infrastructure & & 10 \\
\hline Confidentiality and security concerns & & 9 \\
\hline Lack of technical expertise & & 6 \\
\hline Resistance from patients & & 7 \\
\hline Resistance from administrators & & 4 \\
\hline Resistance from users & & 3 \\
\hline Lack of usefulness of telemedicine & & 3 \\
\hline
\end{tabular}

\section{METHOD}

\section{Clinic Background and Demographics}

The Primary Care Clinic of North Texas offers a variety of services to uninsured adults in the Dallas-Fort Worth (DFW) metroplex area. The clinic has three locations in urban and suburban communities and has several different units. These units include the Pap smear and EKG unit, generic drug unit, arthritis, and lupus treatment programs, information technology (IT), and medical records department (Lintz, 2018). The clinic has faced unprecedented demographic transitions in the last five years. According to the clinic's 2018 patient demographic data, the majority of the population it serves is ethnic minority (62\% Hispanic, 22\% African American, 10\% Native American, and 6\% white). A substantial proportion of the population are undocumented immigrants from Mexico and Latin America. They are often ineligible for privately funded health services, so the outpatient clinic is their primary care provider (Lintz, 2018). Furthermore, the clinic has switched to virtual visits or telemedicine visits during the pandemic. In addition to risk of financial strain from the pandemic, these special challenges have further hindered the providers who are already struggling as a result of low reimbursement rates.

\section{Data Collection}

The researcher obtained a list of primary healthcare providers from the clinic administration in its three locations. A self-administered questionnaire along with the informed consent explaining the study objectives was mailed to all 67 primary healthcare providers at the clinic in May 2020. In addition, respondents were assured confidentiality and free to decline participation in the study. Nonrespondents received telephone calls between July and August of 2020 to remind them to complete the survey. The final survey response was $70 \%$ (47/67). Shown in Table 1 are the characteristics of the respondents.

\section{Measures}

The study questionnaire developed by Primary Care Collaborative (PCC) was utilized to measure perceived barriers and provider characteristics related to telemedicine use (Quick COVID-19 Survey, 2020). The existing instrument used a multistep approach to identify and refine a set of items assessing a primary care provider's ability to meet patients' needs during the pandemic. Many of the items employed a Likert scale response format. Other questions were openended to afford further refinement and clarification of responses about issues related to adoption of telemedicine that had not been included in the survey.

The survey first collected respondent demographic information, practice characteristics, and detailed responses regarding respondents' experience with telemedicine. Survey items comprised two main foci: major barriers to telemedicine adoption and respondent characteristics pertaining to usage of telemedicine. With respect to perceived barriers to telemedicine adoption, respondents were asked the following question: "Based on the list below, to what extent is each of these a potential barrier to telemedicine adoption?" (response options were "not a barrier," "somewhat of a barrier," "moderate barrier," "and "a major barrier"). The barriers included cost of equipment, lack of reimbursement, inadequate technical infrastructure, confidentiality and security, lack of technical expertise, resistance from patients, resistance from administrators, resistance from users, and other-please be specific.

The second set of questions asked respondents the following: "During the last 12 months, have you used telemedicine?" (response options were 1 for "yes" and 2 for "no"). A final set of questions were open-ended: "What are some of the reasons for you to consider using telemedicine in practice?" and "Is there anything else concerning the use or adoption of telemedicine that we have not covered that you believe should be included?”.

\section{RELIABILITY AND VALIDITY}

The existing instrument's items had initially been factor analyzed to establish concurrent and convergent validity and found to be at an adequate level (Etz et al., 2019). Reliability of the scales was established using coefficient alpha (Etz et al., 2019). 
Table 2. Multiple logistic regression results for characteristics associated with telemedicine use in the past 12 months $(n=47)$

\begin{tabular}{|c|c|c|c|c|}
\hline \multirow[b]{2}{*}{ Characteristics } & \multicolumn{4}{|c|}{$\mathbf{C I}^{* * *}$} \\
\hline & AOR* & Low & High & p-value ${ }^{* * * *}$ \\
\hline \multicolumn{5}{|l|}{ Age } \\
\hline$<39$ years & 3.66 & 1.65 & 7.66 & $.002^{\text {***** }}$ \\
\hline \multicolumn{5}{|c|}{ Years of employment } \\
\hline$<5$ years & 4.46 & 1.23 & 8.79 & 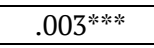 \\
\hline \multicolumn{5}{|c|}{ Subspecialist status } \\
\hline Subspecialist & 3.86 & 1.50 & 5.48 & $.011^{* * * * * *}$ \\
\hline
\end{tabular}

Note. *AOR: Adjusted odds ratio; **CI: Confidence interval; $* * * * 0.05$ Any telemedicine use in past 12 months ( $1=$ yes; $2=$ no): Dependent variable. Respondents' characteristics, including demographic \& practice characteristics: Independent variables

\section{Analysis}

First, the study centered on determining the percentage of respondents reporting that a given issue posed a major barrier to telemedicine adoption.

Second, multiple logistic regression was utilized to assess how respondent characteristics (gender, age, location, years of employment, and specialty status) were associated with telemedicine use in the past 12 months (coded " 1 " if the respondent reported any telehealth usage in the past 12 months and coded "2" for no use). When a study has one nominal and two or more measurement variables, employing multiple logistic regression analysis is appropriate (Adams and Lawrence, 2019).

Third, a chi-square test for independence was conducted to determine the relationship between the number of perceived barriers and telemedicine use. Assumptions for the chi-square independence test include that (1) data are categorical, (2) observations are independent (the same individual is not observed multiple times), (3) groups are mutually exclusive, and (4) expected frequencies have at least five for each categorical variable (Adams and Lawrence, 2019).

Finally, data were analyzed using the Statistical Package for Social Science (SPSS). A p-value $<0.05$ was used as the level for statistical significance.

\section{RESULTS}

\section{Sample Demographics}

Table 1 shows demographic information of respondents. Most respondents were male (60\%). 57\% were younger than 39 years old; $43 \%$, 40 years or older. In addition, $60 \%$ were urban primary healthcare providers, and $40 \%$ were suburban providers. More than one-half had been employed 10 years or more in this clinic. Also, approximately $80 \%$ were primary care providers; the remainder were subspecialist providers.

\section{Perceived Barriers to Adoption of Telemedicine}

As portrayed in Table 1, the most frequently mentioned barriers to telehealth adoption were "cost of equipment" and "lack of reimbursement" (58\% of respondents), followed by "inadequate technical infrastructure" and "confidentiality and security concerns" (19\% of respondents). 6\% of respondents reported hindrances related to a lack of technical expertise and
Table 3. Chi-square independence test results for association between the number of perceived barriers and telemedicine use in the past 12 months $(n=47)$

\begin{tabular}{lcc}
\hline Number of perceived barriers Telemedicine use (\%) & p-value \\
\hline $1-3$ barriers & $21(45 \%)$ & $.028^{*}$ \\
\hline $4-6$ barriers & $18(38 \%)$ & $.0003^{*}$ \\
\hline $7-8$ barriers & $5(11 \%)$ & $.0002^{*}$ \\
\hline $9+$ barriers & $3(6 \%)$ & $.0001^{*}$ \\
\hline Total & $\mathbf{4 7 ( 1 0 0 \% )}$ & \\
\hline$*<05$ & &
\end{tabular}

\section{*p<.05}

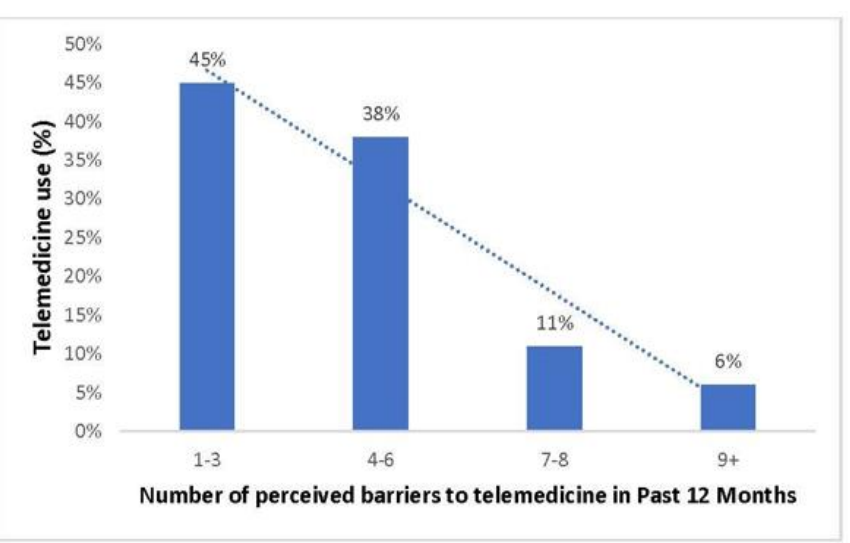

Figure 1. Relationship between the number of perceived barriers and telemedicine use $(n=47)$

support. In addition, $14 \%$ of respondents cited "resistance from patients, administrator, and users to participate in telemedicine" as obstacles. Last, only $3 \%$ of respondents identified "lack of usefulness of telemedicine" as an impediment to telemedicine in their practices.

\section{Provider Characteristics Related to Telemedicine Use}

A multiple logistic regression (Table 2) was performed to ascertain the association between respondents' characteristics and existence of telemedicine use in the past 12 months. Findings revealed that respondents employed fewer than 5 years (adjusted odds ratio $[A O R]=4.46 ; 95 \%$ confidence interval $[\mathrm{CI}]=1.23-8.79 ; \mathrm{p}=.003$ ) and younger than 40 years of age (AOR=3.66; 95\% $\mathrm{CI}=1.65-7.66 ; \mathrm{p}=.002$ ) and working as subspecialists ( $\mathrm{AOR}=3.86$; 95\% $\mathrm{CI}=1.50-5.48 ; \mathrm{p}=.011$ ) were more likely than their counterparts to report telemedicine use in the past 12 months.

\section{Relationship Between Number of Perceived Barriers and Telemedicine Use}

The results of the chi-square independence test (Table 3) revealed that there was a negative relationship between the number of perceived barriers to telemedicine adoption and telemedicine use in the past 12 months. Overall, as respondents perceived more barriers in their practice, they were less likely to be using telemedicine. For example, among those perceiving 9 or more obstacles $(p=.0001)$, only $6 \%$ reported telemedicine usage in the past 12 months. However, among those perceiving only 1 to 3 impediments ( $p=.028), 45 \%$ reported employment of telemedicine in the past 12 months. Provided in Figure 1 is a visualization that manifesting that there was a strong negative relationship between the number of perceived obstructions and telemedicine utilization. 


\section{Analysis of Open-Ended Questions}

Findings from the open-ended questions revealed that $100 \%$ of providers would consider using telemedicine if they were reimbursed adequately. In addition, 57\% expressed that dysfunctional, inept, inflexible administrators within the clinic had failed to offer providers requisite critical resources quickly to foster adoption of telemedicine to treat patients during the pandemic. Moreover, 97\% propounded that a traditional health care system long acclimated to attending to age-old diseases-such as tuberculosis and sexually transmitted disease-was now inadequate for addressing COVID-19 demand. Plus, these providers felt that the absence of a functioning national electronic health information system to support telehealth and health information exchange during the pandemic served as another serious buffer to offering efficacious telehealth medicine.

\section{DISCUSSION}

The study explored major barriers to adoption of telemedicine among primary healthcare providers and characteristics related to telemedicine use during a portion of the pandemic. Study findings indicated that, despite reports of increasing telemedicine access in recent years, major perceived barriers to telemedicine adoption among this research's primary healthcare providers were consistent with those identified in previous germane work (Kruse et al., 2018; Levine et al., 2014; Sisk et al., 2020). A majority of this undertaking's respondents (58\%) reported that the cost of equipment and absence of adequate reimbursement were key impediments to adoption of telemedicine in their clinic. Also, nearly $19 \%$ of respondents identified "inadequate technical infrastructure" and "confidentiality and security concerns" as obstacles. Because reimbursement for telemedicineespecially across state lines-has been largely limited and has been impeded by complexity of the federal and state reimbursement policies, providers may well not be cognizant of or intimately familiar with what regulations to abide by per the U.S. Department of Health and Human Services (HHS) (2016).

Additionally, the results showed that those primary healthcare providers working as subspecialists and employed fewer than 5 years with an age of 40 years and younger were significantly more likely to report telemedicine use than their peer providers. These findings accord with extant work which has found that 40-year old or younger primary healthcare providers practiced some form of telehealth at a higher rate than physicians 45 years of age or older-especially younger subspecialists (2018 Survey of America's Physicians, 2018; Bodenheimer and Pham, 2010; Lintz, 2020).

Furthermore, the current study involved examining the association between the number of perceived barriers and usage of telemedicine. Findings manifested that, as the number of perceived obstacles increased, the likelihood of adoption of telemedicine decreased. The results of this relationship revealed that to encourage providers to incorporate telemedicine in their practice, a range of impediments need to be addressed. Finally, all respondents indicated that they would consider using telemedicine if they were reimbursed adequately; this result signifies that financial issues are especially critical and vexing ones for providers in deciding whether to utilize telemedicine. This investigation's results accord with previous research that has suggested that the lack of supportive leadership and policies and reimbursement coverage for telemedicine services have historically been a major obstruction to telemedicine adoption, especially among providers in primary care practices (Bajowala et al., 2020; Blumenthal et al., 2020; Kruse et al., 2018; Levine et al., 2014; Sisk et al., 2020).

\section{Implications for Healthcare Providers}

The results of this work are instructive to healthcare providers and facilities in the U.S. and around the globe vis-àvis developing a holistic strategic plan of action to address the key barriers in the adoption of telemedicine during the pandemic-especially in primary healthcare settings where health care resources are scarce. Moreover, as healthcare facilities continue to add new features to stay abreast of rapidly changing health care trends, the current COVID-19 outbreak may offer a unique opportunity for healthcare providers and facilities around the world to invest in telemedicine infrastructure for long-term use in response to this crisis while keeping patients and health providers safe during the pandemic (Monaghesh and Hajizadeh, 2020). Therefore, to motivate healthcare providers to utilize telemedicine and to make health information technology (IT) advancements, a variety of administrative, financial, and technical barriers should be remedied through a collaborative effort among healthcare facilities, healthcare providers, policy makers, hospital administrators, and patients.

\section{Limitations and Future Research}

Limitations of this study include the small sample size $(n=47)$. A larger, more dispersed sample could have improved generalizability and offered additional insight into the needs and potential solutions to adoption of telemedicine. In addition, certain survey questions regarding participants' points of view could have been subject to personal interpretation. However, this researcher believes that the results of the current study were instrumental to evaluate barriers to providers' telemedicine use.

In the future, an in-depth study with a larger, variegated sample should be utilized to enhance understanding of the specific process for overcoming the identified barriers to adoption of telemedicine systems. This type of study would help focus on the required resources needed for healthcare providers and facilities across states and regions, as well as countries and hemispheres. Also, focusing on financial incentives and costs involved in providers' usage of telemedicine systems could move the healthcare industry a step toward eliminating these major economic impediments.

\section{CONCLUSIONS}

Since the beginning of the pandemic, many healthcare organizations have transformed healthcare delivery through telemedicine, thus allowing for ongoing medical care while ensuring the safety of patients and health care providers 
(Temesgen et al., 2020). Although much has been written over the past decade about telemedicine-associated benefits and barriers on the quality of care and safety, minimal efforts have systematically assessed the range of potential implementation, technical, financial, and interoperability hurdles in the adoption of telemedicine. The results of the current study indicated that the cost of equipment and lack of adequate reimbursement remain major barriers to incorporating telemedicine into many healthcare providers' practices. Furthermore, these perceived barriers make providers less likely to use telemedicine. Accordingly, providers' and policy makers' efforts are sine qua non for decreasing these obstacles and ultimately encouraging future telemedicine adoption during and after the pandemic.

Author notes: The author agrees with the results and conclusions. Funding: No external funding is received for this article.

Declaration of interest: The author declares that there is no competing interests.

Ethics approval and consent to participate: Not applicable. Availability of data and materials: All data generated or analyzed during this study are available for sharing when appropriate request is directed to corresponding author.

\section{REFERENCES}

2018 Survey of America's Physicians. (2018). The Physicians Foundation by Merritt Hawkins. 1-83. Available at: https://physiciansfoundation.org/wp-content/uploads/ 2018/09/physicians-survey-results-final-2018.pdf (Accessed: 4 March 2021).

Adams, K. A. and Lawrence, E. K. (2019). Research methods, statistics, and applications. Thousand Oaks, CA: SAGE.

Bajowala, S. S., Milosch, J. and Bansal, C. (2020). Telemedicine pays: Billing and coding update. Current Allergy and Asthma Report, 20(10), 60. https://doi.org/10.1007/s11882-02000956-y

Blue, R., Yang, A. I., Zhou, C., et al. (2020). Telemedicine in the era of coronavirus disease 2019 (COVID-19): A neurosurgical perspective. World Neurosurgery, 139, 549557. https://doi.org/10.1016/j.wneu.2020.05.066

Blumenthal, D., Fowler, E. J., Abrams, M. and Collins S. R. (2020). Covid-19-Implications for the health care system. The New England Journal of Medicine, 383(15), 1483-1488. https://doi.org/10.1056/NEJMsb2021088

Bodenheimer, T. and Pham, H. H. (2010). Primary care: Current problems and proposed solutions. Health Affairs, 29(5), 799-805. https://doi.org/10.1377/hlthaff.2010.0026

Chauhan, V., Galwankar, S., Arquilla, B., et al. (2020). Novel coronavirus (COVID-19): Leveraging telemedicine to optimize care while minimizing exposures and viral transmission. Journal of Emergencies, Trauma, and Shock, 13(1), 20-24. Available at: https:/www.ncbi.nlm.nih.gov/pmc/articles/PMC7161346/

CMS. (2020). CMS. Available at: https://www.cms.gov/ newsroom/fact-sheets/medicare-telemedicine-healthcare-provider-fact-sheet (Accessed: 4 March 2021).
CMS. (2020). National Center for Immunization and Respiratory Diseases (NCIRD), Division of Viral Diseases. Available at: https://www.cdc.gov/coronavirus/2019-ncov/hcp/ telehealth.html (Accessed: 9 August 2021).

Etz, R. S., Zyzanski, S. J., Gonzalez, M. M., Reves, S. R., O’Neal, J. P. and Stange, K. C. (2019). A new comprehensive measure of high-value aspects of primary care. Annals of Family Medicine, 17(3), 221-230. https://doi.org/10.1370/ afm.2393

Friedman, D., Rajan, R. and Seidmann, A. (2019). Randomized trial of telemedicine for migraine management. Cephalalgia, 39(12), 1577-1585. https://doi.org/10.1177/ 0333102419868250

Hing, E., Hall, M. J. and Xu, J. (2008). National hospital ambulatory medical care survey: 2006 outpatient department summary. National Health Statistics Report, 4, $1-31$.

Kruse, C., Karem, P., Shifflett, K., Vegi, L., Ravi, K. and Brooks, M. (2018). Evaluating barriers to adopting telemedicine worldwide: A systematic review. Journal of Telemedicine and Telecare, 24(1), 4-12. Available at: https://pubmed.ncbi.nlm.nih.gov/29320966/

Levine, M., Richardson, J. E., Granieri, E. and Reid, M. C. (2014). Novel telemedicine technologies in geriatric chronic non-cancer pain: Primary care providers' perspectives. Pain Medicine, 15(2), 206-213. https://doi.org /10.1111/pme.12323

Lintz, J. (2018). A qualitative study of master patient index (MPI) record challenges from health information management professionals' perspectives. Perspectives in Health Information Management, 1-13.

Lintz, J. (2020). Adoption of computerized information management systems (CIMS) functions: Urban versus rural primary healthcare providers. International Journal of Healthcare Management. https://doi.org/10.1080/ 20479700.2020.1756109

Martin, A. B., Probst, J. C., Shah, K., Chen, Z. and Garr, D. (2012). Differences in readiness between rural hospitals and primary care providers for telemedicine adoption and implementation: Findings from a statewide telemedicine survey. Journal of Rural Health, 28(1), 8-15. https://doi.org/ 10.1111/j.1748-0361.2011.00369.x

Moazzami, B., Razavi-Khorasani, N., Moghadam, A. D., Farokhi, E. and Rezaei, N. (2020). COVID-19 and telemedicine: Immediate action required for maintaining healthcare providers well-being. Journal of Clinical Virology, 126, 104345. https://doi.org/10.1016/j.jcv.2020. 104345

Molfenter, T., Boyle, M., Holloway, D. and Zwick, J. (2015). Trends in telemedicine use in addiction treatment. Addiction Science \& Clinical Practice, 10, 14. https://doi.org/ 10.1186/s13722-015-0035-4

Monaghesh, E. and Hajizadeh, A. (2020). The role of telehealth during COVID-19 outbreak: A systematic review based on current evidence. BMC Public Health, 20, 1193. https://doi.org/10.1186/s12889-020-09301-4

Ohannessian, R., Duong, T. A. and Odone, A. (2020). Global telemedicine implementation and integration within 
health systems to fight the COVID-19 pandemic: A call to action. JMIR Public Health \& Surveillance, 6(2), e18810. https://doi.org/10.2196/18810

Paschall, B. (n. d.). Telehealth flattens, redefines the COVID-19 curve. Available at: https://www.fortherecordmag.com/ news/041720_exclusive.shtml (Accessed: 4 March 2021).

Primary Care Collaborative. (2020). PCC. Available at: https://www.pcpcc.org/2020/04/08/primary-care-covid19-week-4-survey?language=en (Accessed: 4 March 2021).

Quick COVID-19 Survey. (2020). The Larry A. Green Center. Available at: https://www.green-center.org/covid-survey (Accessed: 4 March 2021).

Rahi, S., Khan, M. M. and Alghizzawi M. (2020). Factors influencing the adoption of telemedicine health services during COVID-19 pandemic crisis: An integrative research model. Enterprise Information Systems, 15(6), 769-793. https://doi.org/10.1080/17517575.2020.1850872

Redd, T. K., Doberne, Lattin, D., et al. (2015). Variability in electronic health record usage and perceptions among specialty vs. primary care physicians. AMIA Annual Symposium Proceeding, 2015, 2053-2062.

Sisk, B., Alexander, J., Bodnar, C., et al. (2020). Pediatrician attitudes toward and experiences with telehealth use: Results from a national survey. Academic Pediatrics, 20(5), 628-635. https://doi.org/10.1016/j.acap.2020.05.004
Smith, A. C., Thomas, E. and Snoswell, C. L., et al. (2020). Telehealth for global emergencies: Implications for coronavirus disease 2019 (COVID-19). Journal of Telemedicine and Telecare, 26(5), 309-313. https://doi.org/ 10.1177/1357633X20916567

Temesgen, Z. M., DeSimone, D. C., Mahmood, M., Libertin, C. R., Palraj, B. R. V. and Berbari, E. F. (2020). Health care after the COVID-19 pandemic and the influence of telemedicine. Mayo Clinic Proceedings, 95(9), S66-S68. https://doi.org/ 10.1016/j.mayocp.2020.06.052

U.S. Department of Health and Human Services (HHS). (2016). Report to congress: E-health and telemedicine. ASPE. Available at: https://aspe.hhs.gov/pdf-report/reportcongress-e-health-and-telemedicine (Accessed: 4 March 2021).

World Health Organization (WHO). (2020). WHO DirectorGeneral's opening remarks at the media briefing on COVID19-11 March 2020. Available at: https://www.who.int/ $\mathrm{dg} /$ speeches/detail/who-director-general-s-openingremarks-at-the-media-briefing-on-covid-19---11-march2020 (Accessed: 4 March 2021).

World Health Organization (WHO). (n. d.). Coronavirus disease (COVID-19) pandemic. Available at: https://www.who.int/ emergencies/diseases/novel-coronavirus-2019 (Accessed: 4 March 2021). 